\title{
BMJ Quality Hydration Stickers - Improving oral hydration in vulnerable patients
}

\author{
Alysha Bhatti, Javier Ash, Shyam Gokani, Suveer Singh
}

To cite: Bhatti A, Ash J, Gokani S, et al. Hydration Stickers - Improving oral hydration in vulnerable patients. BMJ Quality Improvement Reports 2017;6:u211657.w6106 doi:10.1136/bmjquality. u211657.w6106
CrossMark

Imperial College School of Medicine and Chelsea and Westminster NHS Trust, United Kingdom

Correspondence to Dr Suveer Singh suveer.singh@ic.ac.uk

\section{ABSTRACT}

Dehydration is a growing problem among elderly patients in hospital wards. Incidents such as those raised in the Francis Report highlight a problem that may not have been sufficiently addressed by current schemes. This improvement project aimed to identify the barriers faced by staff in improving oral hydration and to design and implement an effective solution. A 33 patient pilot study carried out at Chelsea \& Westminster Hospital NHS Trust, United Kingdom, revealed that a significant proportion of patients were reported to be dehydrated on admission, with few having their hydration needs addressed. Staff cited time pressures and unclear task responsibility as the major barriers. The intervention was a Hydration Sticker education scheme. These stickers were placed on patient cups, notes and beside areas as a visual prompt for staff and family members to encourage the patient to drink. The intervention was implemented on the Acute Assessment Unit and Stroke ward through a poster campaign. The Hydration Stickers scheme resulted in a 6.5-fold increase in patients' hydration needs being assessed and addressed. Coupled with the low implementation cost and ease of use, Hydration Stickers may be a simple, effective, transferable and sustainable solution to the problem of dehydration among elderly inpatients.

\section{PROBLEM}

Dehydration secondary to inadequate oral fluid intake contributes to significant morbidity and mortality among patients and is thus a major safety concern. In addition to its negative effect on patient wellbeing and recovery, it is also an economic healthcare burden.

We have seen that the hospital environment is often not conducive to patients achieving an adequate hydration status. Reasons for this include poor access to water, lack of encouragement to increase oral fluid intake and embarrassment of having to visit the toilet frequently. The elderly, who now constitute an increasing proportion of hospital inpatients, are particularly vulnerable to the effects of dehydration. Our study, carried out on the Acute Assessment Unit (AAU) and Stroke ward at Chelsea and Westminster
Hospital NHS Trust (CW), United Kingdom, highlighted the additional barriers to achieving adequate hydration, faced by this patient cohort. CW is a large teaching hospital in Central London with a diverse patient population.

Our study has shown that doctors frequently write "push oral fluids" as part of their management plan. Current NHS schemes to encourage hydration in these patients are limited; but include methods such as coloured cups, regular discussions with the nursing team to focus on encouraging a specific patient to drink, as well as active monitoring of oral fluid intake using intake charts. However there is ineffective implementation of these hydration schemes, as only $14 \%$ of patients with "push oral fluids" in their management plan were subsequently found to be included in hydration schemes. Hence there is a deficiency of effective primary prevention of dehydration in the hospital ward setting.

Our project thus aims to:

1. Investigate the burden of dehydration on the AAU and Stroke ward at Chelsea and Westminster Hospital NHS Trust (CW), United Kingdom

2. Evaluate the barriers encountered by staff in improving oral hydration

3. Formulate and implement a simple, costeffective intervention to improve patients' oral fluid intake on the AAU and Stroke ward at CW, so that by the end of a 3 week period at least $60 \%$ of patients in the study sample are actively included in hydration schemes

\section{BACKGROUND}

Water is an essential nutrient in the maintenance of human health. When net loss of body water exceeds input, dehydration occurs. Inadequate hydration has detrimental effects on inpatients; predisposing to kidney stones, pressure ulcers, hypotension, poor glycaemic control and thromboembolic events such as coronary heart disease and stroke. ${ }^{1}$ 
Furthermore, the financial saving that could result from tackling this problem effectively is estimated to be around $£ 0.95$ billion with a reduction of 83,000 bed days per annum. ${ }^{2}$

Awareness of the problem has grown due to the Mid Staffordshire scandal in which an estimated 1200 patient deaths occurred due to lack of provision of basic care. Anecdotal reports described patients drinking from flower vases due to the inaccessibility of drinking water. The Francis Report, an objective inquiry into the scandal, detailed "even water or the means to drink it could be hard to come by", concluding that "the arrangements and best practice for providing food and drink to patients require constant review, monitoring and implementation." 3

The iatrogenic nature of the problem is further supported by the National Reporting and Learning System study, which identified the leading cause of dehydration in an 8 year period of patient safety incidents, to be caused by a "failure to act." 3

Current systems in place to optimise nutritional needs for vulnerable patients include the "red tray" scheme. This involves delivering meals to nursing-dependent patients (for example, those who are malnourished or have difficulty swallowing) on red trays, which acts as a visible indicator of the assistance they require during meal times and a reminder to the nursing staff to accurately document their food intake. ${ }^{4}$ Whilst useful for identifying nutritional risk, there are currently no quality improvement schemes at CW which are designed to specifically improve the oral fluid intake of vulnerable patients.

Thus regular assessment of patients' hydration status and implementation of appropriate interventions to tackle dehydration are paramount in ensuring patient safety.

\section{BASELINE MEASUREMENT}

Our first objective was to investigate the burden of dehydration on the AAU and Stroke ward at CW. In order to quantify this assessment, we reviewed and analysed clinical notes from 33 adult patients on the day of their admission. Patients were deemed to be dehydrated if the clinical examination revealed signs of dry mouth, poor skin turgor, delayed capillary refill time and those who were oliguric (urine output $<0.5 \mathrm{ml} / \mathrm{kg}$ /hour) or anuric (no urine output). The results revealed that 9 out of the 33 patients $(27 \%)$ audited were dehydrated on admission.

Having shown that dehydration was indeed a problem, we then collected further data in order to ascertain how clinicians responded to the problem. We found that clinicians' primary response to tackling the burden of dehydration was to include phrases such as "push oral fluids" in the patient's management plan. Our study showed that 7 out of the $33(21 \%)$ of patients' management plans in the AAU and Stroke ward included similar wording, whilst 2 out of the 33 patients were deemed to be nil-by-mouth. Of the patients we identified with "push oral fluids" written in their management plans, only $14 \%$ were found to be actively included in hydration schemes. We ascertained whether patients were actively included in a hydration scheme by interviewing the speech and language therapist, dietician and nurse (s) responsible for each of the dehydrated patients.

Our next objective was to understand the barriers encountered by members of the multidisciplinary team (MDT) in improving oral hydration. A process study using semi-structured interviews was conducted with 15 members of the MDT on the Stroke ward (1 consultant, 3 registrars, a senior house officer, 5 nurses, 2 members of the catering staff, 2 dieticians and a physiotherapist). During the semi-structured interviews, suggestions of barriers were allowed to arise organically as opposed to being chosen from a pre-determined list. This method was chosen in order to prevent limitation in the diversity of comments and to minimise any potential external influences. A medical student member of the Quality Improvement team identified barriers by extracting keywords from the data through simple observation. The results showed that $47 \%$ of staff cited the greatest barriers to achieving adequate hydration status of their patients to be that of time pressures whilst discontinuity of patient care with diffuse task responsibility accounted for the second most common reason (Figure 1).

\section{DESIGN}

Once the problem had been analysed and assessed, a solution was formulated to comply with the six dimensions of healthcare quality. ${ }^{5}$ With the current financial burden on the NHS, a long-term solution to tackle the dehydration problem would need to be inexpensive and easily reproducible to ensure a high implementation and compliance rate. Furthermore, the scheme would need to be sustainable in order to meet the objectives and demonstrate continued efficacy.

A Quality Improvement team was thus assembled consisting of a senior clinical lead, junior clinical staff,

Staff Barriers To Increasing Patient Oral Fluid Intake

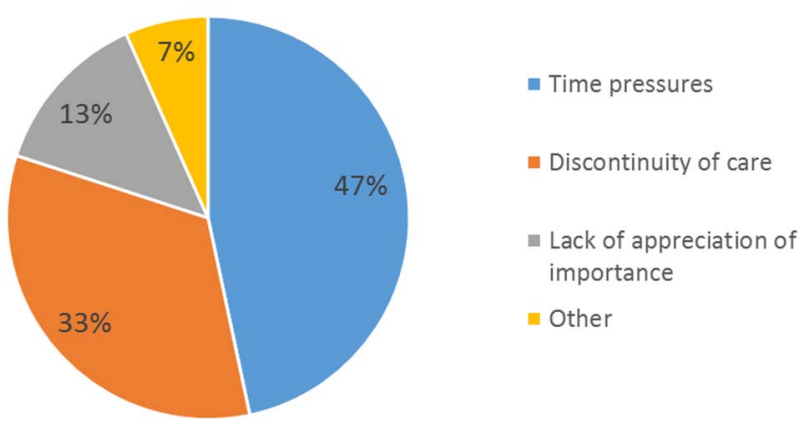

Figure 1 Staff Barriers To Increasing Patient Oral Fluid Intake 
medical students and nurse representatives. A Hydration Stickers scheme was conceived to improve oral hydration through the use of disposable Hydration Stickers for patient bedside areas, patient notes and cups (Figure 2). Responsibility for placing the stickers would lie with all members of staff on the ward, including doctors, nurses, healthcare assistants, physiotherapists, dieticians and occupational therapists. The stickers would serve as a clear visual prompt for family members and the wider MDT alike to encourage oral fluid intake. The use of a disposable adhesive sticker is a simple and sustainable tool which aims to more effectively communicate the intention behind phrases such as "push oral fluids", which are often included in the management plans of dehydrated patients.

Lack of awareness of the stickers was predicted to be a potential barrier to the successful implementation of the scheme. Therefore, the Quality Improvement team developed posters highlighting the Hydration Stickers scheme that would be placed in key locations on the AAU and Stroke ward. Hydration Stickers were promoted to staff via the poster campaign, during MDT meetings, as well as through verbal encouragement on the AAU and Stroke Ward. Success of the intervention was measured over 3 weeks.

Figure 2 Hydration Stickers

Poster

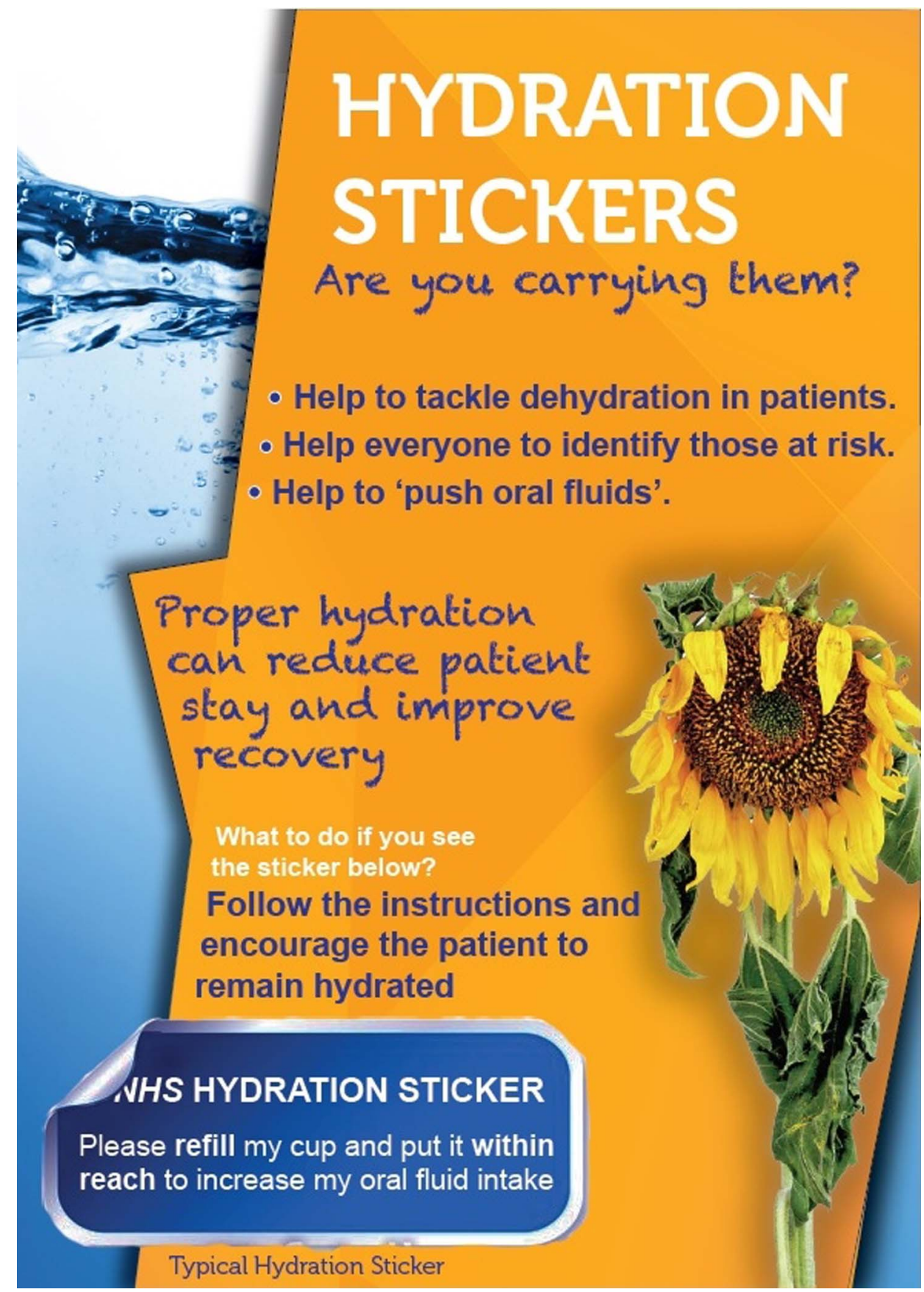




\section{STRATEGY}

Our SMART aim was to improve patients' oral fluid intake on the AAU and Stroke ward at CW so that by the end of a 3 week period, at least $60 \%$ of patients in the study sample were actively included in hydration schemes. A mixed methodology approach, in which three 'Plan Do Study Act' (PDSA) cycles were undertaken, was adopted to achieve our aim.

PDSA cycle 1: The baseline measurements made on the point prevalence sample of 33 patients on the AAU and Stroke ward of CW, highlighted the burden of dehydration. These results were consistent with our prediction, since the increasing number of elderly inpatients are more susceptible to becoming dehydrated. An intervention-based strategy was thus devised. Specific points were addressed; most importantly, to overcome lack of formal procedures for enforcing hydration schemes, and the time constraints faced by healthcare professionals, which emerged as key themes during the semi-structured interviews undertaken as baseline measurements. A campaign was conceived to increase oral hydration through the use of disposable Hydration Stickers for patient bedside areas, patient notes and patient cups. These would serve as visual prompts to increase patients' oral fluid intake. A poster designed to highlight the new scheme was placed in common areas on the AAU and Stroke ward (Figure 3) and 10-15 Hydration Stickers were distributed to doctors on both wards. Uptake of the scheme was monitored by way of observation on both wards of the number of patients who had been enrolled in the Hydration Sticker scheme. Patients were classed as enrolled if at least one Hydration Sticker had been affixed to their cup or surroundings at the time of their afternoon meal. Over the course of the following week, no further verbal contact or prompts were given. At the end of the one week implementation period, $86 \%$ of patients audited were found to be actively included in hydration schemes.

PDSA cycle 2: After one week of the scheme being implemented, feedback from the doctors was that

\section{Impact of Hydration Stickers scheme on patient hydration}

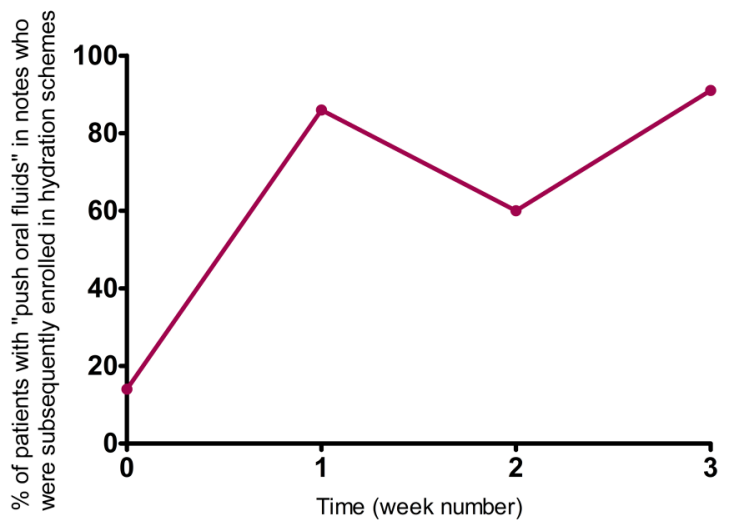

Figure 3 Impact of Hydration Stickers scheme on patient hydration greater awareness of the scheme could be achieved by informing more members of the MDT. Thus the Hydration Sticker scheme was formally introduced at the weekly MDT meeting. Attendees included doctors, nurses, dieticians, physiotherapists, speech and language therapists and occupational therapists. Attendees were educated about the scheme and how to enact it as well as being provided with an information booklet and 10-15 Hydration Stickers. After one week, $60 \%$ of patients audited were found to be actively included in hydration schemes. This did not meet our expectations and demonstrated that a more sustainable solution was required to ensure that momentum for the scheme did not dissipate prematurely.

PDSA cycle 3: Given the slight reduction in the uptake of the scheme after PDSA cycle 2, a 15 minute focus group was held with doctors from the wards in which barriers to implementation and potential improvements to the scheme were explored. Suggestions of barriers were allowed to arise organically and were identified by extracting keywords from the data through simple observation.

One issue which emerged was that of poor continuity of care, and thus we hypothesised that our change idea (intervention) would be more successful if the responsibility for the Hydration Sticker scheme was assigned to a particular individual. Thus two named Hydration Officers were appointed on each ward and an information booklet containing their details was circulated to staff. One nurse and one junior doctor were selected to be Hydration Officers based on their enthusiasm for the scheme and the duration of their post on the ward. Their role was to keep a stock of Hydration Stickers so that they were easily accessible to staff, ensure that new staff were aware of the scheme and answer any queries regarding the scheme. Responsibility for placing the stickers still lay with the whole MDT. After one week, $91 \%$ of patients audited were found to be actively included in hydration schemes. This improvement confirmed our hypothesis that assigning active responsibility for the intervention to individuals on the ward would increase the sustainability of the scheme.

\section{RESULTS}

The success of the Hydration Stickers initiative was evaluated after 3 weeks of its implementation on the AAU and Stroke Ward at CW. This enabled us to determine the level of uptake by MDT members and to assess the primary outcome. The primary outcome was the percentage of patients originally identified as having "push oral fluids" documented in their notes who were subsequently included in hydration schemes.

Our baseline measurements showed that only $14 \%$ of patients in our study were actively included in hydration schemes. Introduction of the Hydration Sticker scheme resulted in a six-fold increase in patients' hydration needs being addressed (86\%) after one week.

However measurements from the subsequent week showed a $26 \%$ reduction in patients' hydration needs 
being addressed despite the scheme being formally introduced to more staff members at the weekly MDT meeting.

The final post-implementation audit at the end of 3 weeks showed that $91 \%$ of patients' hydration needs were being addressed (Figure 4).

\section{LESSONS AND LIMITATIONS}

Dehydration among inpatients has a substantial impact on clinical outcomes; leading to prolonged bed days, failed discharges and ultimately an increase in mortality. 6

Various factors account for a failure among patients to consume an adequate amount of water during their hospital stay. This may be due to limited access to drinking facilities, medication effects, or embarrassment of having to visit the toilet frequently. It is crucial that the medical team encourages and facilitates appropriate water consumption in these patient groups to aid recovery and minimise morbidity. Hence there is a clear need to readdress how a patient's hydration status is communicated to all those involved in their care.

The main barrier faced by hydration schemes is due to the time pressures on staff which hinders their ability to implement and act on the scheme in order to improve the patient's hydration status. Furthermore, the lack of continuity of care compounded by a rise in agency staff and changing rotas, means that despite some members of the MDT being aware of a patient's sub-optimal hydration status, there is no clear delegation of task responsibility.

This study has evaluated the use of Hydration Stickers on patient notes, cups and bedside areas as a solution to this problem. Hydration Stickers are inexpensive to produce and their disposable nature means that they constitute a non-invasive and low-risk approach, thus ensuring safe use across patient groups. Through their ease of use and cost efficacy, Hydration Stickers thus represent a sustainable solution to overcome these barriers as well as empowering friends and family members to recognise and address the patient's hydration needs.

By collecting qualitative feedback from staff members regarding their views on the scheme and holding regular focus groups where potential improvements could be explored, we were able to continually optimise the scheme with the aim of increasing its efficacy.

Limitations to this study include the small sample size and potential selection bias. This means that it is not possible to translate the results to other patient groups with absolute certainty. Additionally, the intervention is only likely to be beneficial, so long as patients with a fluid restriction order or specific electrolyte concerns are excluded. Furthermore, the time constraints of this study meant that a limited number of PDSA cycles were achieved which precluded the ability to undertake statistical analysis of our results. Moreover, this study relied on the accuracy of patient notes to determine the patient hydration status and their inclusion in active hydration schemes. Due to the inherent inaccuracy of patient notes, the study may have underestimated the proportion of patients suffering from dehydration in the audit group. Lastly, the study examined and evaluated the qualitative impact of the intervention on hydration status, rather than taking a quantitative approach that would yield more accurate results.

In future, more PDSA cycles should be implemented which would allow us to measure a sustained change in hydration status of elderly inpatients. Quantification of hydration status using electrolyte measurements and urine output as well as monitoring progress with Statistical Process Charts would provide a more reliable measure of intervention success and allow us to assess the impact of balancing measures such as fluid overload states.

Furthermore, after discussions with the cleaning staff, the use of semi-adhesive stickers was advised. This would lead to less frustration by staff when trying to remove the stickers but also enable stickers to be displayed in a greater variety of sites in the patient bay, thus enhancing the efficacy of the scheme and contributing to its ongoing sustainability. Lastly, whilst the Hydration Sticker scheme was introduced to staff at the weekly MDT meeting, a formal introduction in further MDT meetings as well as grand rounds would widen the scope to the whole hospital and ensure that staff are aware of what it entails and how to enact the scheme. One must be vigilant to the possibility however, that increasing the number of patients enrolled in the scheme through the suggested optimisation procedures, may not actually reduce rates of dehydration.

This study was primarily focussed on the elderly population as they represent the largest burden of dehydration in the hospital. However, as the problem is also rife among other patient cohorts, we envisage that the Hydration Sticker scheme will be trialled in a variety of clinical settings in order to increase its reach and benefit.

It is also worth considering that this intervention only has the potential to be effective for those patients who have already been identified as dehydrated. However, inherent inaccuracies of the physical examination and initial assessment of patients mean that the true burden of dehydration may be underestimated. Implementing interventions such as the Hydration Sticker scheme should go hand-in-hand with improving the identification of patients who are dehydrated. Future work in this area is required, and could focus on the value of more rigorous assessments such as urine electrolytes. ${ }^{7}$

\section{CONCLUSION}

The results show that dehydration is an ongoing problem that is often addressed simply by writing "push oral fluids" in the patient management plan. These plans are not always communicated to other members of staff and hence patients' hydration needs are often not addressed. 
The Hydration Sticker scheme increased staff awareness of patient hydration status and gained traction by the MDT, thus achieving the project's initial aim. There was a 6.5-fold increase in the number of patients having their hydration needs recognised by the end of the 3 week implementation period; showing that Hydration Stickers used as a visual prompt are an effective tool in assisting staff and relatives to encourage patients to increase oral fluid intake. By appointing Hydration Officers on the wards who reinforce the goals of the scheme, the Quality Improvement team has taken effort to ensure sustainability of the scheme.

Additional avenues for further development and optimisation include the use of semi-adhesive stickers, quantification of patient hydration status and formal introductions to the scheme at further MDT meetings and grand rounds. Such improvements are likely to enhance the efficacy of the scheme, making it more amenable to be translated to a wider patient cohort in the hospital setting and ensuring its ongoing sustainability.

Acknowledgements We would like to thank Chelsea \& Westminster Imperial College NHS Trust and in particular Dr Suveer Singh and Dr Rula Najim for their insight and support.
Declaration of interests Nothing to declare.

Ethical approval Ethical approval was not required for this project as work was carried out as a service improvement.

Open Access This is an open-access article distributed under the terms of the Creative Commons Attribution Non-commercial License, which permits use, distribution, and reproduction in any medium, provided the original work is properly cited, the use is non commercial and is otherwise in compliance with the license. See:

- $h t t p: / / c r e a t i v e c o m m o n s . o r g / l i c e n s e s / b y-n c / 2.0 /$

- http://creativecommons.org/licenses/by-nc/2.0/legalcode

\section{REFERENCES}

1. Popkin B, D'Anci K, Rosenberg I. Water, hydration, and health. Nutrition Reviews 2010;68:439-458.

2. Campbell N. Dehydration: why is it still a problem? Nursing Times 2011;107:12-15.

3. Lecko C. Patient safety and nutrition and hydration in the elderly. A Report by The Health Foundation 2013.

4. Bradley $L$, Rees $C$. Reducing nutritional risk in hospital: the red tray. Nursing Standard. 2003;17:33-37.

5. Committee on Quality of Health Care In America. Crossing the Quality Chasm: A New Health System for the 21st Century. Washington, DC: National Academy Press; 2001. 6 p.

6. NHS England. Guidance - Commissioning Excellent Nutrition and Hydration 2015-2018. A Report by NHS England; 2015.

7. McGee S, Abernethy W, Simel D. Is This Patient Hypovolemic? JAMA 1999;281:1022-1029. 